\title{
Influence of Electric and Magnetic Fields on Dielectric Response of Oil-Based Ferrofluid
}

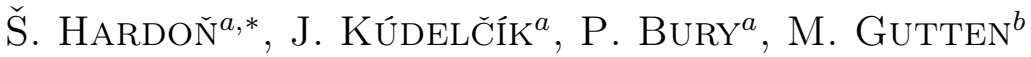 \\ ${ }^{a}$ Department of Physics, ${ }^{b}$ Department of Measurement and Applied Electrical Engineering University of Žilina, \\ Univerzitná 1, 01026 Žilina, Slovakia
}

\begin{abstract}
The changes in dielectric parameters and structural arrangement of transformer oil based ferrofluid with magnetic nanoparticles upon the effect of an external magnetic field and an electric field were studied by dielectric spectroscopy. The frequency dependence of dissipation factor was measured within the frequency range from 1 $\mathrm{mHz}$ to $10 \mathrm{kHz}$ by a capacitance method. In the presence of the magnetic field the interaction between the magnetic field and magnetic moments of nanoparticles led to the aggregation of nanoparticles. The electric field had also effects on the change in the electric dipole moment of particles by transfer of counterions. The observed low frequency relaxation maximum of dissipation factor was explained by Schwarz theory of electric double layer polarization. The shift of this maximum with the intensity of electric was caused by change of relaxation time of the nanoparticles-counterions systems.
\end{abstract}

DOI: 10.12693/APhysPolA.133.477

PACS/topics: 47.65.Cb, 77.22.Gm, 77.22.Ch, 75.50.Mm, 75.75.Jn

\section{Introduction}

Nowadays the magnetic liquids or ferrofluids are considered as new technological materials which are of enormous interest for applications. Ferrofluids are defined as a stable colloidal suspensions of solid nanoparticles (size is in range $1-100 \mathrm{~nm}$ ) in essence, which are conventional base fluids most often as oils, water or glycols. During the preparation of ferrofluids, the particles are uniformly dispersed throughout the liquid volume. The acoustic spectroscopy is also one of useful tool for the study of structure changes of ferrofluids in the magnetic fields [1]. Among then belongs successful application of thermosphysical property of ferrofluid in audio technique. The next wide applications are in transformers, where thermal conductivity and convective heat transfer properties of ferrofluid are higher as classical oil so their also improve the operating parameters of power transformers [2]. Moreover, it was publish that the transformer oil with nanoparticles can increase the electrical breakdown strength [3].

The aim of the study of dielectric spectroscopy is correctly recognize the mechanism of dielectric relaxation in ferrofluids. These dielectric relaxations can be seen in the frequency dependence of dissipation factor. The low and high frequency maximas should be clearly observed [4,5]. On the basis of Maxwell-Wagner model was estimated that one maximum of $\tan \delta$ appear at hundreds of $\mathrm{MHz}$ frequency, which exhibit in many colloidal systems containing different dielectric constituents. The next low frequency relaxation process, occurring in ferrofluids is described by the Schwarz model $[6,7]$. This

*corresponding author; e-mail: hardon@fyzika.uniza.sk model supposes the creation of the electric double layers (EDLs) due to the presence the space charge in the ferrofluid. EDLs are created on the particle surfaces as a result of electrostatic interactions between the particles and the space charge. An application of electric field leads to a polarization of EDLs and this process is correlated with a corresponding relaxation process, which is responsible for the low-frequency dielectric behavior of this system [5]. The third observed effect is caused by the accumulation of the ion impurities at the electrode interfaces, known as the electrode polarization (EP) [4]. The EP causes an anomalous increase in the complex dielectric permittivity in a lower frequency region by many orders of magnitude.

The dielectric behaviour of ferrofluids changes with the application of a magnetic field and with the relative orientation of the magnetic and electric fields. This phenomena is studied using a magnetodielectric anisotropy. The dependence of dielectric parameters of a ferrofluid on the applied of magnetic field is called magneto-dielectric effect, and this effect is studied by many researchers. This effect describes the increase or decrease values of relative permittivity in parallel or perpendicular fields [8, 9].

\section{Materials and methods}

For measurements we used a ferrofliud based on transformer oil (MOGUL TRAFO CZ-A, Paramo) and magnetite nanoparticles $\mathrm{Fe}_{3} \mathrm{O}_{4}$. As a surfactant was used oleic acid to the stabilization of magnetic colloidal nanoparticles. The density of ferrofluid was $865 \mathrm{~kg} / \mathrm{m}^{3}$ and the mean diameter of the magnetite nanoparticles was $7.9 \pm 2.4 \mathrm{~nm}$ with the volume concentration $0.45 \%$. The DC magnetic susceptibility 0.06 and saturation magnetization of the ferrofluid was $2.33 \mathrm{~A} \mathrm{~m}^{2} \mathrm{~kg}^{-1}$.

Commercial liquid crystal (LC) cells as capacitors to achieve more precise dielectric measurement of ferrofluids in our experiment we have used. The cells are composed 
of two transparent flat glass (sandwiched type) substrates coated with Indium Tin Oxide (ITO) conductive layers acting as electrodes. The transparent ITO layers allowed us to check the homogenous filling of the cell. Liquid crystal cells with two parallel plate ITO electrodes, whose distance apart $d=5 \mu \mathrm{m}$. The area of active electrode was $30 \mathrm{~mm}^{2}$, were used as capacitors (sample holders). The capacity of the empty cell was $C_{0}=56 \mathrm{pF}$.

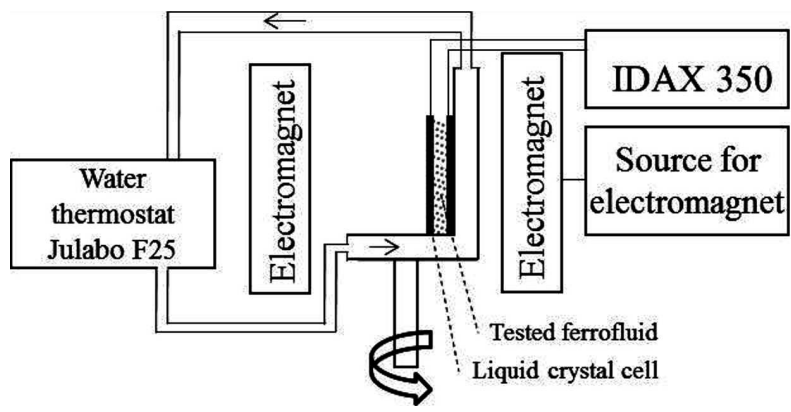

Fig. 1. Experimental arrangement of measurement.

The experimental arrangement is shown in Fig. 1. The LC filled with ferrofluid was thermally boned to the holder of sample, which allows rotation of capacitor with holder in the external magnetic field generated by electromagnet. The temperature of the experimental sample and holder during the measurement was controlled by water thermostat JULABO F25 with accuracy $0.1^{\circ} \mathrm{C}$. The measurements were carried out at the temperature of $25^{\circ} \mathrm{C}$. Dissipation factor, real and imaginary capacitances by IDAX 350 in the frequency range from $10 \mathrm{mHz}$ to $10 \mathrm{kHz}$ were measured at different electric field intensities, achieved by varying the potential difference on the capacitor electrodes. These dielectric parameters also at parallel and at perpendicular orientation of magnetic field to the electric field were measured.

\section{Experimental results and discussion}

The frequency dependence of dissipation factor measured in the range from $0.01 \mathrm{~Hz}$ to $10 \mathrm{kHz}$ is depicted on Fig. 2. The low frequency relaxation maximum is clearly observed, because this relaxation process is the result of the ferrofluid properties. To explain the low frequency relaxation process can be described by the Schwarz model. In this figure we can also observe the magneto-dielectric effect of studied ferrofluid. When the external magnetic field was applied the whole development of the dissipation factor shifted to left side. The frequency dependence of dissipation factor in parallel orientated fields is only a function of the electric field. For the perpendicular orientation of the electric field to the magnetic field, the Lorentz force has a maximum effect on the drift of the nanoparticles in the direction of the electric field. This force causes the motion of nanoparticles in a spiral, which causes longer tracks of the nanoparticles in the system and it is connected with the increase of dissipation factor. The position of the local maximum of $\tan \delta$ is also dependent on the orientation of the magnetic field with respect to the electric field, for parallel orientation the maximum is at the frequency of $20 \mathrm{~Hz}$ and for perpendicular $17 \mathrm{~Hz}$. The change of dissipation factor with application of magnetic field can be resulting from a rearrangement of nanoparticles to new structures, as oligomers or chains. The change of structural arrangement of magnetic nanoparticles in studied ferrofluids by magnetic field was confirmed by acoustic measurement [1]. These agglomeration higher have electric dipoles so generate higher depolarization field, which further reduces the total intensity of electric field. Consequently, the relaxation maximum shifts towards lower frequencies with the application of magnetic field.

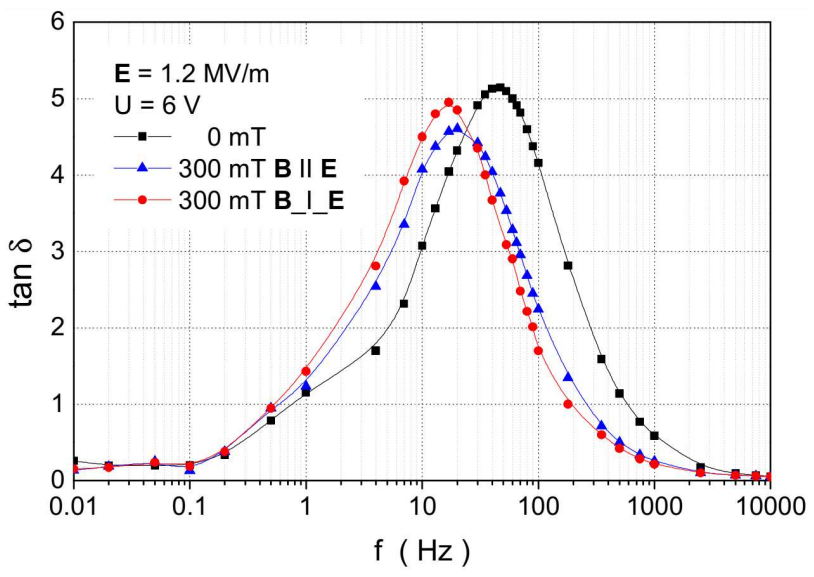

Fig. 2. The effect of the external magnetic field and its orientation in the regard to the electric field on the dissipation factor.

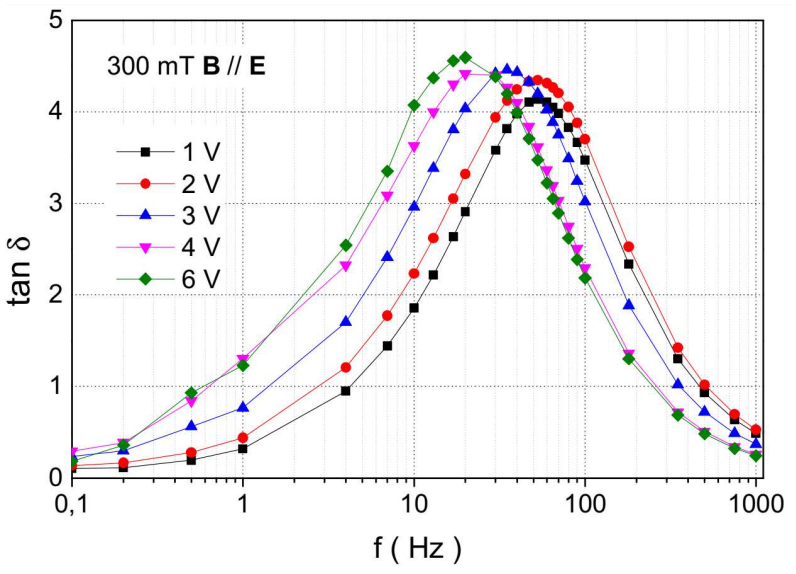

Fig. 3. The frequency dependence of dissipation factor at different electric field intensities, achieved by varying the potential difference on the capacitor electrodes.

Figure 3 shows the frequency dependence of dissipation factor at different electric field and its parallel orientation to static magnetic field $300 \mathrm{mT}$. In the case of perpendicular orientations of fields the effect of different electric field is same but shifted to lower frequencies. The change of the intensity of electric field by varying the voltage from range $1 \mathrm{~V}$ to $6 \mathrm{~V}$ applied to the capacitor was done. Similar results are presented in work [10]. 


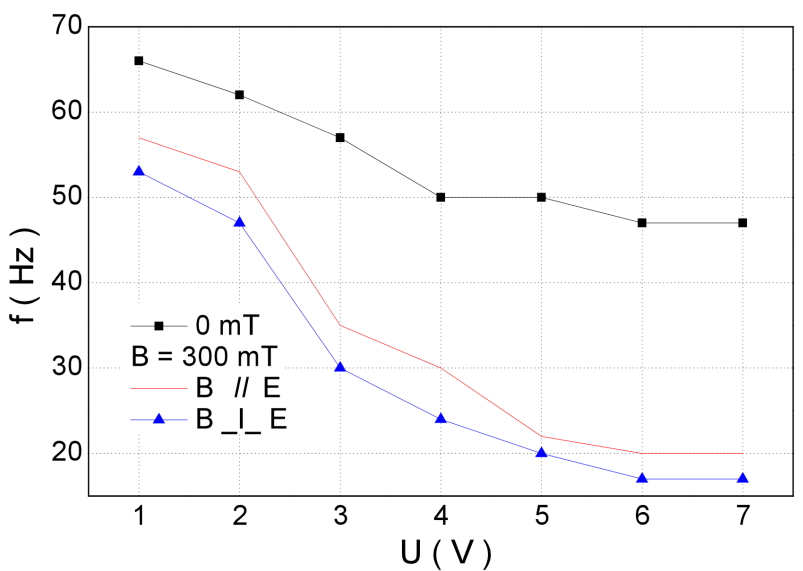

Fig. 4. The value of relaxation frequency maximum depending on the applied intensity of electric field without, parallel and perpendicular of magnetic field.

In the Fig. 4 we can observe the values of frequency maximum's of dissipation factor for various intensities of electric field without and with parallel or perpendicular orientation of the magnetic field $300 \mathrm{mT}$, respectively. With the increasing of the intensity of electric field, there is the decrease of the frequency of relaxation maximum of $\tan \delta$. In whole range of intensities of electric field the relaxation frequency maximum is higher for $E \| B$ than for $E \perp B$ configuration. For the lower magnetic field $100 \mathrm{mT}$ the similar results were acquired, but all frequencies are shifted to higher values and all measured values also for $200 \mathrm{mT}$ are summarized in the Table I.

TABLE I

Dependence of the value of relaxation frequency maximum for dissipation factor from the electric field from voltage range at $1 \mathrm{~V}$ to $7 \mathrm{~V}$ at different orientation of magnetic to electric fields.

\begin{tabular}{c|c|c|c|c}
\hline \hline \multirow{2}{*}{$\begin{array}{c}\text { AC } \\
\text { voltage }\end{array}$} & \multicolumn{4}{|c}{ Frequency [Hz] } \\
\cline { 2 - 5 } & \multicolumn{2}{|c|}{ at $100 \mathrm{mT}$} & \multicolumn{2}{|c}{ at $200 \mathrm{mT}$} \\
\cline { 2 - 5 } & $\|$ & $\perp$ & $\|$ & $\perp$ \\
\hline $1 \mathrm{~V}$ & 62 & 55 & 60 & 53 \\
$2 \mathrm{~V}$ & 55 & 49 & 53 & 47 \\
$3 \mathrm{~V}$ & 37 & 32 & 35 & 30 \\
$4 \mathrm{~V}$ & 32 & 27 & 30 & 25 \\
$5 \mathrm{~V}$ & 22 & 19 & 20 & 17 \\
$7 \mathrm{~V}$ & 22 & 19 & 20 & 17
\end{tabular}

The decrease of the relaxation frequency maximum of the dissipation factor with the increase of intensity of the electric field can be caused by two effects [5]. In the first case, the magnetite particles are in contact and EDL is destroyed at the contact region of the particles. Consequently, the counterions are moved by an electric field on the surface of this agglomeration, so it looks like a particle having a larger size. This fact yields to a larger value of relaxation time and therefore to a smaller value of the relaxation frequency. In the second case, the magnetic dipolar interaction is responsible for particle ag- glomeration and is much stronger than the repulsive electrostatic interaction, then counterions from the exterior layer are be forced to remain in the vicinity region of particles. Consequently, the mobility of these counterions is smaller, yielding to a larger value of the relaxation time and again to a smaller value of corresponding of relaxation frequency. So if the maximum of the dissipation factor decreases to a smaller frequency values, then it can be suppose that the particle agglomerations are present within the ferrofluid.

\section{Conclusions}

The low frequency relaxation maximum of dissipation factor was observed and described by the Schwarz model of electric double layer polarization. With the increase of electric field the development of dissipation factor shifts to lower frequencies. At three different values of external magnetic field the magnetodielectric effect in ferrofluid was confirmed in high electric fields $0.2-1.4 \mathrm{MV} / \mathrm{m}$. The decrease of low frequency maximum at application of the external magnetic field could be explained by aggregation of nanoparticles and its next decreases with the intensity of the electric field is caused by transfer of counterions within given arrangement.

\section{Acknowledgments}

This work was supported by the project VEGA $1 / 0510 / 17$.

\section{References}

[1] J. Kúdelčík, P. Bury, J. Drga, P. Kopčanský, V. Závišová, M. Timko, Acta Phys. Pol A 121, 1169 (2012).

[2] J. Liu, L. Zhou, G. Wu, Y. Zhao, IEEE Trans. Dielec. Electrical Insul. 19, 510 (2012).

[3] J. Kúdelčik, P. Bury, P. Kopčanský, M. Timko, Physics procedia 9, 78 (2010).

[4] M. Rajňák, B. Dolník, J. Kurimský, R. Cimbala, P. Kopčanský, M. Timko, J. Chem. Phys. 146 014704 (2017).

[5] I. Malaescu, C. N. Marin, J. Colloid Interface Sci. 251, 73 (2002)

[6] G.A. Schwarz, J. Chem. Phys. 66, 2636 (1962).

[7] J. Miao, M. Dong, M. Ren, X. Wu, L. Shen, H. Wang, J. Appl. Phys 113, 204103 (2013).

[8] M. Timko, P. Kopčanský, M. Molcan, L. Tomčo, K. Marton, S. Molokac, P. Rybar, F. Stoian, S. Holotescu, A. Taculescu, Acta Phys. Pol. A 121, 1253 (2012).

[9] P. Zukowski, T.N. Koltunowicz, V. Bondariev, A.K. Fedotov, J.A. Fedotova, J. Alloys Comp. 683, 62 (2016).

[10] M. Rajňák, J Kurimský, B. Dolník, K. Marton, L. Tomčo, A. Taculescu, L. Vekas, J. Kováč, I. Vavra, J. Tothová, P. Kopčanský, M. Timko, J. Appl. Phys. 114, 034313 (2013). 